\title{
PERFIL BIOQUÍMICO RENAL DEL COCODRILO DE TUMBES (Crocodylus acutus) CRIADO EN CAUTIVERIO EN EL NORTE DEL PERÚ
}

\author{
Renal Biochemistry Profile of Kidney in the Tumbes Crocodile \\ (Crocodylus acutus) Reared in Captivity in the North of Peru
}

\author{
Verónica Horikawa K. ${ }^{\text {, }}$ Olga Lí E. ${ }^{1,3}$, César Gavidia C. ${ }^{2}$, Luis Hoyos S. ${ }^{1}$
}

\section{Resumen}

El objetivo del estudio fue determinar el perfil bioquímico renal del cocodrilo de Tumbes (Crocodylus acutus) criado en cautiverio en Tumbes, Perú. Los animales fueron contenidos mediante sujeción manual y se colectaron muestras de sangre $(6 \mathrm{ml})$ mediante punción del seno venoso post-occipital a 60 animales machos (24 juveniles, 27 subadultos y 9 adultos). El valor de ácido úrico fue de $74.2 \pm 25.0 \mathrm{mg} / \mathrm{L}$, creatinina: $1.49 \pm 0.39$ $\mathrm{mg} / \mathrm{L}$, calcio: $10.8 \pm 2.4 \mathrm{mg} / \mathrm{dl}$ y fósforo: de $5.3 \pm 2.0 \mathrm{mg} / \mathrm{dl}$. Se encontró diferencia estadística $(\mathrm{p}<0.05)$ entre juveniles y sub-adultos para el ácido úrico y creatinina, y entre adultos frente a juveniles y sub-adultos para el calcio. No hubo diferencia significativa entre valores de fósforo por grupo etario.

Palabras clave: Crocodylidae, Crocodylus acutus, bioquímica renal

\section{AbSTRACT}

The aim of the study was to determine the biochemistry profile of the kidney in the Tumbes crocodile (Crocodylus acutus) reared in captivity in the north of Peru (Tumbes). Blood samples $(6 \mathrm{ml})$ by puncture of the post-occipital venous sinus were collected to 60 males (24 juveniles, 27 sub-adults, and 9 adults). All animals were manually restrained. Values for uric acid was $74.2 \pm 25.0 \mathrm{mg} / \mathrm{L}$, creatinine $1.49 \pm 0.39 \mathrm{mg} / \mathrm{L}$, calcium $10.8 \pm 2.4$ $\mathrm{mg} / \mathrm{dl}$, and phosphorus $5.3 \pm 2.0 \mathrm{mg} / \mathrm{dl}$. There was significant difference $(\mathrm{p}<0.05)$ between juveniles and sub-adults for uric acid and creatinine, and adults versus younger animals for calcium, whereas phosphorus values were not affected by age.

Key words: Crocodile, Crocodylus acutus, renal profile

\footnotetext{
${ }^{1}$ Laboratorio de Patología Clínica, ${ }^{2}$ Laboratorio de Medicina Veterinaria Preventiva, Facultad de Medicina Veterinaria, Universidad Nacional Mayor de San Marcos, Lima

${ }^{3}$ E-mail: olgalie@hotmail.com
} 


\section{INTRODUCCIÓN}

El cocodrilo de Tumbes (Crocodylus acutus) es una especie amenazada de extinción, incorporada en el Apéndice I de CITES (Convención Internacional para el Comercio de Especies Amenazadas de Fauna y Flora Silvestre) en 1979. El comercio internacional con animales y productos de esta especie está prohibido, pero aun así, las poblaciones naturales no logran recuperar su tamaño y distribución (Thorbjarnarson et al., 2006).

En Perú, desde 1950, bajo la Resolución Suprema N. ${ }^{\circ} 345$, se prohíbe la caza de Crocodylus acutus en su hábitat natural (Escobedo y Mejía, 2003). Desde setiembre de 1996, un zoocriadero del Fondo Nacional de Desarrollo Pesquero (FONDEPES) se dedica a la reintroducción y repoblación con fines comerciales a mediano y largo plazo del C. acutus en la cuenca del río Tumbes y, de esta manera, evitar su exterminio en el país (Pérez et al., 2005).

La bioquímica sanguínea en reptiles está influenciada por la especie, edad, sexo, estado nutricional, estación climática y estado fisiológico, lo cual dificulta la interpretación de los resultados (Campbell, 1996; Barboza et al., 2006; Thrall et al., 2006). No existen reportes completos del perfil bioquímico renal para $C$. acutus en el Perú, de allí que el presente estudio tuvo por objetivo la determinación de los valores bioquímicos renales de ácido úrico, creatinina, calcio y fósforo, a fin de compararlos con los niveles séricos referenciales sanguíneos de crocodílidos.

\section{Materiales y Métodos}

La toma de muestras se realizó en agosto de 2008 en el Zoocriadero del Centro de Acuicultura «La Tuna Carranza», de FONDEPES, ubicado en Puerto Pizarro, departamento de Tumbes, Perú. La zona tenía una temperatura media diaria de $25.5{ }^{\circ} \mathrm{C}$ y una humedad relativa media diaria de $90.7 \%$
(SENAMHI, 2009). Las muestras se procesaron en el Centro de Investigación de Cisticercosis de Tumbes y en el Laboratorio de Patología Clínica de la Facultad de Medicina Veterinaria de la Universidad Nacional Mayor de San Marcos, Lima.

Se trabajó con un tamaño de muestra de 60 cocodrilos criados en cautiverio, siguiendo el teorema del límite central (Daniel, 1996; Martínez, 2001). De estos, 24 fueron juveniles (menos de $90 \mathrm{~cm}$ ), 27 subadultos (entre $90-180 \mathrm{~cm}$ ) y 9 adultos machos (más de $180 \mathrm{~cm}$ ). Los animales estaban clínicamente sanos y fueron criados en similares condiciones de alojamiento, alimentación y manejo. El alimento, a base de hígado, pollo y pescado, era proporcionado entre $1 \mathrm{y}$ 2 veces por semana a los subadultos y adultos, y tres veces por semana a los juveniles, quienes además recibían un suplemento alimenticio.

Se empleó la sujeción manual para la contención física de los animales. La muestra de sangre $(6 \mathrm{ml})$ se obtuvo por punción del seno venoso post-occipital, en tubos al vacío sin anticoagulante, y transportados en refrigeración al laboratorio para la extracción del suero.

Se determinaron los valores séricos de acido úrico (método enzimático), creatinina (método colorimétrico), calcio (método colorimétrico) y fósforo (método UV) utilizando kit comerciales de Wiener Lab. La lectura de la absorbancia se realizó en un espectofotómetro (UV Photometro 4010 Mamhein Boehringer).

Los valores bioquímicos renales se evaluaron mediante estadística descriptiva, empleando la media aritmética como medida de tendencia central y como medida de dispersión se utilizó la desviación estándar. Se utilizó el análisis de varianza para comprobar diferencias estadísticas entre grupos etarios y la prueba de Bonferroni para la comparación de medias (Daniel, 1996). 
Cuadro 1. Valores séricos (promedio \pm d.e.) de bioquímica renal para el cocodrilo de Tumbes (Crocodylus acutus) según grupo etario

\begin{tabular}{lccc}
\hline & Juveniles & Sub-Adultos & Adultos \\
\hline Ácido úrico $(\mathrm{mg} / \mathrm{L})$ & $85.0 \pm 26.6^{\mathrm{a}}$ & $64.1 \pm 19.5^{\mathrm{b}}$ & $75.8 \pm 24.9^{\mathrm{a}, \mathrm{b}}$ \\
Creatinina $(\mathrm{mg} / \mathrm{L})$ & $1.64 \pm 0.43^{\mathrm{a}}$ & $1.31 \pm 0.36^{\mathrm{b}}$ & $1.59 \pm 0.16^{\mathrm{a}, \mathrm{b}}$ \\
Calcio $(\mathrm{mg} / \mathrm{dL})$ & $10.3 \pm 1.8^{\mathrm{a}}$ & $10.5 \pm 2.7^{\mathrm{a}}$ & $13.3 \pm 0.9^{\mathrm{b}}$ \\
Fósforo $(\mathrm{mg} / \mathrm{dL})$ & $5.5 \pm 1.8^{\mathrm{a}}$ & $5.4 \pm 2.0^{\mathrm{a}}$ & $4.57 \pm 2.91$ \\
\hline
\end{tabular}

a,b Superíndices diferentes dentro de filas indican diferencia estadí stica $(p<0.05)$

\section{Resultados}

Los valores de ácido úrico, creatinina, calcio y fósforo fueron de $74.2 \pm 25.0 \mathrm{mg} / \mathrm{L}$, $1.49 \pm 0.39 \mathrm{mg} / \mathrm{L}, 10.8 \pm 2.4 \mathrm{mg} / \mathrm{dl}$ y $5.3 \pm$ $2.0 \mathrm{mg} / \mathrm{dl}$, respectivamente. Se encontró diferencia estadística entre juveniles y sub-adultos en ácido úrico y creatinina $(\mathrm{p}<0.05)$ y entre adultos con los animales jóvenes para el caso del calcio $(\mathrm{p}<0.05)$. Por otro lado, no hubo diferencia significativa por grupo etario para los niveles de fósforo sérico (Cuadro 1).

\section{Discusión}

Los únicos valores de perfil bioquímico renal para Crocodylus acutus encontrados en la literatura científica han sido reportados por el Internacional Species Information System (2002), siendo de $41.0 \pm 21.0 \mathrm{mg} / \mathrm{L}$, $0.49 \pm 0.3 \mathrm{mg} / \mathrm{L}, 12.8 \pm 4.0 \mathrm{mg} / \mathrm{dl}$ y $5.2 \pm 1.7$ $\mathrm{mg} / \mathrm{dl}$ para ácido úrico, creatinina, calcio y fósforo, respectivamente, sin especificar edad, sexo, manejo, ni distribución geográfica. Los valores de calcio y fósforo fueron similares a los encontrados en el presente estudio, pero los valores de ácido úrico y creatinina fueron mayores a los reportados por ISIS.

Estas tendencias fueron similares cuando se compararon los valores de Crocodylus acutus sub-juveniles del presente estudio con animales del mismo grupo etario de las espe- cies Caiman latirostris y Caiman yacare, criados en cautiverio en el noreste argentino (Coppo et al., 2006). Estas diferencias pueden deberse a la ingesta de alimentos con alto contenido proteico (Campbell, 1996). Además, los caimanes jóvenes poseen un patrón mixto de excreción nitrogenada (ureotélico-uricotélico), de allí que el porcentaje de excreción de ácido úrico sea más reducido en estos animales (Coppo et al., 2006).

Las variaciones en los valores de creatinina, calcio y fósforo entre estudios pueden deberse, además, a factores extrínsecos como condiciones ambientales, método de captura, alimentación y métodos de laboratorio utilizados (Campbell, 1996).

La mayor cantidad de ácido úrico en los juveniles frente a los subadultos puede deberse a la alimentación especial que reciben los animales durante este periodo crítico de crianza, con suplementos con vitaminas, minerales y harina de hueso (Fontanillas et al., 1999). En el presente estudio, los juveniles eran alimentados con una mayor frecuencia que los subadultos.

Es posible que los cocodrilos subadultos estuvieran pasando por un periodo de estrés o falta de adaptación al cautiverio, lo que conllevaría a una menor ingesta de alimentos y, por lo tanto, una pobre ganancia de masa muscular. Se conoce que en casos de atrofia muscular severa se reduce la cantidad de creatinina formada, ya que esta es un pro- 
ducto nitrogenado no proteico del metabolismo muscular (Meyer et al., 1998). Asimismo, el mayor contenido de calcio en cocodrilos adultos puede deberse a que sus requerimientos de calcio son menores, de allí que el calcio sérico se presenta en relación a la proporción que ocurre entre osteogénesis y osteolisis (Troiano, 1991).

\section{Conclusiones}

- Se describió el perfil bioquímico renal del cocodrilo de Tumbes (Crocodylus acutus) criado en cautiverio en el norte del Perú.

- Los valores de ácido úrico y creatinina fueron superiores en cocodrilos juveniles en comparación con los cocodrilos sub-adultos.

- Los valores de calcio fueron superiores en cocodrilos adultos en comparación con los cocodrilos más jóvenes.

- Los valores de fósforo fueron similares entre grupos etarios.

\section{Literatura Citada}

1. Barboza N, Mussart N, Ortiz L, Prado W, Coppo J. 2006. Influencia de la especie, sexo, edad, alimentación y temperatura ambiental sobre los electrolitos séricos de caimanes autóctonos. Universidad Nacional del Nordeste. Comunicaciones Científicas y Tecnológicas 2006. Resumen: V-005. [Internet], [16 febrero 2009]. Disponible en: http:// www.unne.edu.ar/Web/cyt/cyt2006/04Veterinarias/2006-V-005.pdf

2. Campbell T. 1996. Clinical pathology. In: Mader D. Reptile medicine and surgery. Philadelphia: WB Saunders. $p$ 252-254.

3. Coppo JA, Mussart NB, Barboza NN, Fioranelli SA, Koza GA, Prado WR. 2006. Physiological changes in serum glucidic and nitrogenic analytes from captive Argentine authoctonous caimans. Rev Vet 17: 103-108.
4. Daniel W. 1996. Bioestadística: base para el análisis de las ciencias de la salud. $5^{\mathrm{a}}$ ed. México: Uthea. $878 \mathrm{p}$.

5. Escobedo A, Mejía F. 2003. El «Cocodrilo de Tumbes» (Crocodylus acutus Cuvier 1807): Estudio preliminar de su estado actual en el norte de Perú. Ecología Aplicada 20(1): 133-135.

6. Fontanillas JC, García C, De Gaspar L. 1999. Los reptiles: biología, comportamiento y patología. Barcelona: MundiPrensa. $130 \mathrm{p}$.

7. [ISIS] International Species Information System. 2002. Reference ranges for physiological data values of American Crocodile (Crocodylus acutus), USA. [Internet], [23 febrero 2009]. Disponible en: http:// www.isis.org.com

8. Martínez M. 2001. Bioestadística amigable. Madrid: Díaz de Santos. 94 p.

9. Meyer Denny J, Meyer Dennis J, Harvey JW. 1998. Veterinary laboratory medicine: interpretation $\&$ diagnosis. $2^{\text {nd }}$ ed. UA: WB Saunders. 373 p.

10. Pérez O, Carrión Z, Luján A. 2005. Avances de la crianza en cautiverio de Crocodylus acutus "Cocodrilo Americano». Informe Técnico. Lima: FONDEPES. $34 \mathrm{p}$.

11. [SENAMHI] Servicio Nacional de Meteorología e Hidrología. 2009. Boletín climático nacional enero 2009. [Internet], [20 febrero 2009]. Disponible en: http://www.senamhi.gob.pe/ $? \mathrm{p}=0701$

12. Thrall MA, Baker DC, Campbell TW, De Nicola D, Fettman MJ, Lassen ED, Rebar A, Weiser G. 2006. Veterinary hematology and clinical chemistry. Iowa, USA: Blackwell. 521 p.

13. Troiano JC. 1991. Manejo sanitario de reptiles en cautiverio. Buenos Aires: Prensa Veterinaria. $176 \mathrm{p}$.

14. Thorbjarnarson J, Mazzotti E, Sanderson F, Buitrago M, Lazcano K, Minkowski M, Muñiz P, Ponce L, et al. 2006. Regional habitat conservation priorities for the American crocodile. Biol Conservation 128:25-36. 